\title{
HYPOELLIPTIC SYSTEMS OF COMPLEX VECTOR FIELDS
}

\author{
H. M. MAIRE
}

Section de Mathématiques, Université de Genève

Case postale 240, CH-1211 Genève 24, Switzerland

0. Introduction. Let $X_{1}, \ldots, X_{2 n}$ be $C^{\infty}$ real vector fields on $\Omega$ open in $\mathbb{R}^{N}$ and put

$$
L_{j}=\frac{1}{2}\left(X_{j}+i X_{j+n}\right), \quad 1 \leq j \leq n, L=\left(L_{1}, \ldots, L_{n}\right) .
$$

The following hypotheses are assumed throughout:

(H1) (Hörmander condition) the brackets of length at most $r$ of the $X_{j}$ generate $T_{x} \Omega, \forall x \in \Omega$;

(H2) $\quad d_{x \xi} X(x, \xi)$ are linearly independent;

(H3) $\quad\left[L_{j}, L_{k}\right]=0, \forall j, k \in[1, n]$.

Under (H1), it is well known that the system $\left\{X_{j}\right\}_{1 \leq j \leq 2 n}$ is $(1-1 / r)$ subelliptic, i.e.

$$
\|u\|_{1 / r}^{2} \leq C\left(\sum_{j=1}^{2 n}\left\|X_{j} u\right\|^{2}+\|u\|^{2}\right), \quad u \in C_{\mathrm{c}}^{\infty}(\Omega),
$$

cf. Bolley-Camus-Nourrigat [1]. In particular, for $\omega$ open $\subset \Omega$ we have

$$
u \in \mathcal{D}^{\prime}(\Omega), \quad X_{j} u \in C^{\infty}(\omega), 1 \leq j \leq 2 n \Rightarrow u \in C^{\infty}(\omega) .
$$

Problem. Give geometric conditions to guarantee $L$ to be hypoelliptic:

$$
u \in \mathcal{D}^{\prime}(\Omega), \quad L_{j} u \in C^{\infty}(\omega), 1 \leq j \leq n \Rightarrow u \in C^{\infty}(\omega) .
$$

Examples. 0) $N=2 n,\left(x_{1}, \ldots, x_{n}, y_{1}, \ldots, y_{n}\right) \in \Omega, X_{j}=\partial / \partial x_{j}, X_{n+j}=$ $\partial / \partial y_{j}, L_{j}=\partial / \partial \bar{z}_{j}$. Then $L$ is the Cauchy-Riemann operator, $r=1$ and $L$ is elliptic.

1) $N=2 n+1,\left(x_{1}, \ldots, x_{n}, y_{1}, \ldots, y_{n}, t\right) \in \Omega, X_{j}=\partial / \partial x_{j}-y_{j} \partial / \partial t, X_{n+j}=$ $\partial / \partial y_{j}+x_{j} \partial / \partial t, r=2$. Here, $L$ is the induced Cauchy-Riemann operator on the hypersurface $M=\left\{\left.\left(z_{0}, \ldots, z_{n}\right) \in \mathbb{C}^{n+1}\left|\operatorname{Re} z_{0}=\frac{1}{2}\right| z_{1}\right|^{2}+\ldots+\frac{1}{2}\left|z_{n}\right|^{2}\right\}$; it is not hypoelliptic because when taking Fourier transforms in $t$, we get 
$\widehat{L}_{j}=\bar{\partial}_{z_{j}}-\tau z_{j} / 2=e^{\tau|z|^{2} / 2} \bar{\partial}_{z} e^{-\tau|z|^{2} / 2}$ and for $\widehat{u}(z, \tau)=e^{\tau|z|^{2} / 2}$ if $\tau<0,=0$ if $\tau>0$, it is clear that $L u=0$ but $u \notin C^{\infty}$.

2) Same as above but $M$ is defined by the equation $\operatorname{Re} z_{0}=-\frac{1}{2}\left|z_{1}\right|^{2}-\ldots-$ $\frac{1}{2}\left|z_{p}\right|^{2}+\frac{1}{2}\left|z_{p+1}\right|^{2}+\ldots+\frac{1}{2}\left|z_{n}\right|^{2}, 0<p<n$. In this case, $L$ satisfies

$$
\sum_{j=1}^{2 n}\left\|X_{j} u\right\|^{2} \leq C\left(\sum_{j=1}^{n}\left\|L_{j} u\right\|^{2}+\|u\|^{2}\right), \quad u \in C_{\mathrm{c}}^{\infty}(\Omega)
$$

(cf. [3]) and is, therefore, hypoelliptic.

\section{Maximal hypoellipticity}

R e mark. It is out of the question to characterize hypoellipticity for general systems like Nirenberg and Treves [8] did for $n=1$. Even a characterization of $\delta$-subellipticity:

$$
\|u\|_{1-\delta}^{2} \leq C\left(\sum_{j=1}^{n}\left\|L_{j} u\right\|^{2}+\|u\|^{2}\right), \quad u \in C_{\mathrm{c}}^{\infty}(\Omega),
$$

seems beyond the scope of present techniques, except for $n=1$ which is the Egorov-Hörmander theorem [4].

Definition. The system $L$ is called maximal hypoelliptic in $\Omega$ if (1) holds. It is maximal hypoelliptic at $\left(x_{0}, \xi_{0}\right) \in T^{*} \Omega \backslash 0$ if $\sum_{1}^{n}\left\|\Psi X_{j} \cdot u\right\|^{2} \leq C\left(\sum_{1}^{n}\left\|L_{j} u\right\|^{2}+\right.$ $\left.\|u\|^{2}\right), u \in C_{\mathrm{c}}^{\infty}(\Omega)$, where $\Psi$ is an elliptic pseudodifferential operator at $\left(x_{0}, \xi_{0}\right)$.

Let $\Sigma=\left\{(x, \xi) \in T^{*} \Omega \backslash 0 \mid X_{j}(x, \xi)=0,1 \leq j \leq 2 n\right\}$ be the characteristic set of $X_{1}, \ldots, X_{2 n}$ and for $(x, \xi) \in \Sigma$ define the Levi matrix $\mathcal{L}(x, \xi)$ by $\mathcal{L}_{j k}(x, \xi)=$ $i\left\{L_{j}, \bar{L}_{k}\right\}(x, \xi), 1 \leq j, k \leq n$, where $\{$,$\} is the Poisson bracket. Denote by$ $\lambda_{1}(x, \xi), \ldots, \lambda_{n}(x, \xi) \in \mathbb{R}$ the eigenvalues of $\mathcal{L}(x, \xi)$.

TheOREM (Nourrigat [9]). The system $L$ is maximal hypoelliptic at $\left(x_{0}, \xi_{0}\right)$ if and only if there exists a neighbourhood $U$ of $\left(x_{0}, \xi_{0}\right)$ and $C>0$ such that

$\mathrm{D}(0)$

$$
\max \left(\left|\lambda_{1}\right|, \ldots,\left|\lambda_{n}\right|\right) \leq C \max \left(0, \lambda_{1}, \ldots, \lambda_{n}\right) \quad \text { in } U \text {. }
$$

Comments. 1) Sufficiency of $\mathrm{D}(0)$ was proved in [6] when $L=\bar{\partial}_{\mathrm{b}}$.

2) In the non-degenerate case, i.e., $\lambda_{1} \neq 0, \ldots, \lambda_{n} \neq 0$ everywhere, $\mathrm{D}(0)$ is equivalent to the condition $\mathrm{Y}(0)$ of Folland-Kohn [2].

3) The theorem covers the subellipticity result of Egorov-Hörmander [4] provided we add $\sum\left|X_{j}(x, \xi)\right|$ on the right hand side of $\mathrm{D}(0)$.

4) The proof relies on a general theorem by Helffer-Nourrigat [3] which reduces maximal hypoellipticity to injectivity in $\mathcal{S}\left(\mathbb{R}^{2 n}\right)$ of operators with polynomial coefficients.

2. Case of $(0, q)$-forms for $\bar{\partial}_{\mathrm{b}}$. From now on, we consider the induced Cauchy-Riemann operator on a real hypersurface of $\mathbb{C}^{n}$; therefore, we may 
suppose that locally

$$
L_{j}=\frac{\partial}{\partial \bar{z}_{j}}+i \frac{\partial f / \partial \bar{z}_{j}}{1+i \partial f / \partial t} \partial t
$$

with $f: \Omega \rightarrow \mathbb{R}$ of class $C^{\infty}, \Omega$ open $\subset \mathbb{C}^{n} \times \mathbb{R}$. Then $L$ gives the $\bar{\partial}_{\mathrm{b}}$ complex

$$
0 \longrightarrow \Lambda^{0,0} C^{\infty}(\Omega) \stackrel{L^{0}}{\longrightarrow} \Lambda^{0,1} C^{\infty}(\Omega) \stackrel{L^{1}}{\longrightarrow} \ldots \stackrel{L^{n-1}}{\longrightarrow} \Lambda^{0, n} C^{\infty}(\Omega) \longrightarrow 0,
$$

where $\Lambda^{0, q} C^{\infty}(\Omega)=\left\{\sum_{|J|=q} u_{J} d \bar{z}_{J} \mid u_{J} \in C^{\infty}(\Omega)\right\}$ and

$$
L^{q}\left(\sum_{|J|=q} u_{J} d \bar{z}_{J}\right)=\sum_{|J|=q, 1 \leq j \leq n} L_{j} u_{J} d \bar{z}_{j} \wedge d \bar{z}_{J} .
$$

To $\bar{\partial}_{\mathrm{b}}$ we associate its formal adjoint complex $\bar{\partial}_{\mathrm{b}}^{*}$ :

$$
0 \longrightarrow \Lambda^{0, n} C^{\infty}(\Omega) \stackrel{L^{n-1 *}}{\longrightarrow} \Lambda^{0, n-1} C^{\infty}(\Omega) \longrightarrow \ldots \stackrel{L^{0 *}}{\longrightarrow} \Lambda^{0,0} C^{\infty}(\Omega) \longrightarrow 0 .
$$

R e mark. The following general result by Kohn [5] on $L^{2}$ complexes:

$$
L^{q-1} L^{q-1 *}+L^{q *} L^{q} \text { subelliptic } \Rightarrow \operatorname{ker} L^{q}=\operatorname{im} L^{q-1}
$$

shows that regularity implies solvability.

Definition. The operator $\bar{\partial}_{\mathrm{b}}$ is maximal hypoelliptic on $(0, q)$-forms if

$\left\|\operatorname{Re} L^{q} u\right\|^{2}+\left\|\operatorname{Im} L^{q} u\right\|^{2} \leq C\left(\left\|L^{q} u\right\|^{2}+\left\|L^{q-1 *} u\right\|^{2}+\|u\|^{2}\right), \quad u \in \Lambda^{0, q} C_{\mathrm{c}}^{\infty}(\Omega)$, where $\operatorname{Re} L^{q}, \operatorname{Im} L^{q}$ are defined as in (2) with $X_{j}, X_{n+j}$ on the right hand side.

EXAMPLE. When the Levi matrix $\mathcal{L}$ is non-degenerate, then $\bar{\partial}_{\mathrm{b}}$ is maximal hypoelliptic on $(0, q)$-forms if and only if the condition $\mathrm{Y}(q)$ holds, i.e. the index of $\mathcal{L}$ is different from $q$ and $n-q$ (cf. Folland-Kohn [2], and [3]).

Theorem. Suppose $M=\left\{z \in \mathbb{C}^{n+1} \mid \operatorname{Re} z_{0}=f\left(z_{1}, \ldots, z_{n}\right)\right\} \ni 0$ and the Levi matrix degenerates only at the origin. Then $\bar{\partial}_{\mathrm{b}}$ is maximal hypoelliptic on $(0, q)$-forms if and only if $\mathrm{Y}(q)$ holds on $M \backslash\{0\}$.

The necessity is due to Helffer-Nourrigat [3]. For sufficiency we know (cf. [7]) that the sublevel sets of any localized polynomial of $f$ at 0 have the same homology as those of a quadratic form of index $\neq q$ and $\neq n-q$. It is then possible to solve in $\mathcal{S}\left(\mathbb{R}^{2 n}\right)$ the equation $\widetilde{L}^{q-1} v=u, v \in \Lambda^{0, q-1} \mathcal{S}\left(\mathbb{R}^{2 n}\right)$, where $\widetilde{L}$ is the operator associated to any localized polynomial of $f$, provided $\widetilde{L}^{q} u=0$. If, moreover, $\widetilde{L}^{q-1 *} u=0$, then $u=0$. This proves the injectivity of $\left(\widetilde{L}^{q-1 *}, \widetilde{L}^{q}\right)$ in $\mathcal{S}\left(\mathbb{R}^{2 n}\right)$. Maximal hypoellipticity follows from [3].

Rem a rk. Our proof certainly generalizes.

\section{Hypersurfaces with Levi form having an isolated singularity}

Remark. Only the non-degenerate case and some weakly pseudoconvex cases, e.g. $M=\left\{z \in \mathbb{C}^{n+1} \mid \operatorname{Re} z_{0}=\left(\left\|z_{1}\right\|^{2}+\ldots+\left\|z_{n}\right\|^{2}\right)^{k}\right\}$, were previously known examples of hypersurfaces satisfying the hypothesis of our theorem. 
In order to get more examples we first restrict ourselves to homogeneous real polynomials with non-vanishing hessian; more precisely, let

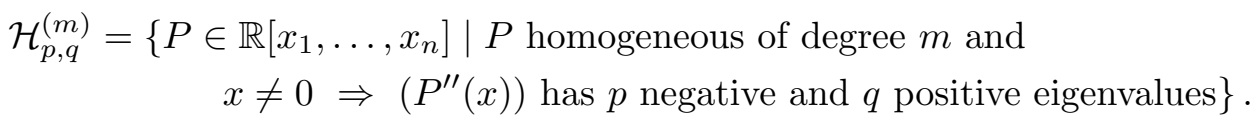

The following results are proved in [7].

Proposition. If $P \in \mathcal{H}_{p, q}^{(m)}$ and $n=p+q \geq 3$ then the map $P^{\prime}: \mathbb{R}^{n} \backslash 0 \rightarrow \mathbb{R}^{n} \backslash 0$ is a diffeomorphism.

Proposition. For $k \geq 1$ and $n=p+q \geq 2$ we have

a) $\mathcal{H}_{p, q}^{(2 k+1)} \neq \emptyset \Leftrightarrow p=q=1$;

b) $\mathcal{H}_{p, q}^{(2)} \neq \emptyset, \forall p, \forall q$;

c) $\mathcal{H}_{p, q}^{(4)} \neq \emptyset \Leftrightarrow p=q=1$ or $p=0$ or $q=0$;

d) $\mathcal{H}_{p, q}^{(2 k)} \neq \emptyset, \forall k \geq 3, \forall p, \forall q$.

ExAmple. $M=\left\{z \in \mathbb{C}^{n+1} \mid \operatorname{Re} z_{0}=-\left(\left|z_{1}\right|^{2}+\ldots+\left|z_{p}\right|^{2}\right)^{k+1}+\varepsilon\left(\left|z_{1}\right|^{2}+\right.\right.$ $\left.\ldots+\left|z_{p}\right|^{2}\right)^{k}\left(\left|z_{p+1}\right|^{2}+\ldots+\left|z_{n}\right|^{2}\right)-\varepsilon\left(\left|z_{1}\right|^{2}+\ldots+\left|z_{p}\right|^{2}\right)\left(\left|z_{p+1}\right|^{2}+\ldots+\left|z_{n}\right|^{2}\right)^{k}+$ $\left.\left(\left|z_{p+1}\right|^{2}+\ldots+\left|z_{n}\right|^{2}\right)^{k+1}\right\}$ is strictly $p$-pseudoconcave and $q$-pseudoconvex away from the origin if $k \geq 2$ and $\varepsilon<2 / k^{2}$.

\section{References}

[1] P. Bolley, J. Camus et J. Nourrigat, La condition de Hörmander-Kohn pour les opérateurs pseudodifférentiels, Comm. Partial Differential Equations 7 (1982), 187-221.

[2] G. B. Folland and J. J. Kohn, The Neumann Problem for the Cauchy-Riemann Complex, Ann. of Math. Stud. 75, Princeton Univ. Press, Princeton 1972.

[3] B. Helffer et J. Nourrigat, Hypoellipticité maximale pour des opérateurs polynômes de champs de vecteurs, Progr. Math. 58, Birkhäuser, 1985.

[4] L. Hörmander, The Analysis of Linear Partial Differential Operators IV, Springer, Berlin 1985.

[5] J. J. Kohn, Differential Complexes, Séminaire de Math. Sup., Univ. de Montréal, 1972.

[6] H. M. Maire, Necessary and sufficient conditions for maximal hypoellipticity of $\bar{\partial}_{\mathrm{b}}$, in: Lecture Notes in Math. 1324, Springer, Berlin 1988, 178-185.

[7] —, Polynômes homogènes réels avec gradient à singularité isolée, Enseign. Math., to appear.

[8] L. Nirenberg and F. Treves, On local solvability of linear partial differential equations I, Comm. Pure Appl. Math. 23 (1970), 1-38.

[9] J. Nourrigat, Systèmes sous-elliptiques II, Invent. Math. 104 (1991), 377-400. 